\title{
Dimensionless parameterization of lidar for laser remote sensing of the atmosphere and its application to systems with SiPM and PMT detectors
}

\author{
Ravil Agishev, ${ }^{1, *}$ Adolfo Comerón, ${ }^{2}$ Alejandro Rodriguez, ${ }^{2}$ and Michaël Sicard ${ }^{2}$ \\ ${ }^{1}$ Kazan National Research Technical University, 10, K. Marx St., 420111 Kazan, Russia \\ ${ }^{2}$ Department of Signal Theory \& Communications, Universitat Politècnica de Catalunya, \\ Jordi Girona 1-3, 08034 Barcelona, Spain \\ ${ }^{*}$ Corresponding author: ravil_agishev@mail.ru
}

Received 10 January 2014; revised 31 March 2014; accepted 1 April 2014;

posted 3 April 2014 (Doc. ID 198759); published 12 May 2014

\begin{abstract}
In this paper, we show a renewed approach to the generalized methodology for atmospheric lidar assessment, which uses the dimensionless parameterization as a core component. It is based on a series of our previous works where the problem of universal parameterization over many lidar technologies were described and analyzed from different points of view. The modernized dimensionless parameterization concept applied to relatively new silicon photomultiplier detectors (SiPMs) and traditional photomultiplier (PMT) detectors for remote-sensing instruments allowed predicting the lidar receiver performance with sky background available. The renewed approach can be widely used to evaluate a broad range of lidar system capabilities for a variety of lidar remote-sensing applications as well as to serve as a basis for selection of appropriate lidar system parameters for a specific application. Such a modernized methodology provides a generalized, uniform, and objective approach for evaluation of a broad range of lidar types and systems (aerosol, Raman, DIAL) operating on different targets (backscatter or topographic) and under intense sky background conditions. It can be used within the lidar community to compare different lidar instruments. (c) 2014 Optical Society of America

OCIS codes: (010.0010) Atmospheric and oceanic optics; (040.0040) Detectors; (080.0080) Geometric optics; (120.0120) Instrumentation, measurement, and metrology; (140.0140) Lasers and laser optics; (280.0280) Remote sensing and sensors.

http://dx.doi.org/10.1364/AO.53.003164
\end{abstract}

\section{Introduction}

When analyzing issues of the lidar system development, maintenance, and upgrading, the problem is posed of how to compare and estimate potential performance of existing and newly developed lidars with design approaches [1, -11$]$, which are sometimes very different. It is clear that lidar designs vary widely depending on specific application, technical

$1559-128 \mathrm{X} / 14 / 153164-12 \$ 15.00 / 0$

(C) 2014 Optical Society of America specifications and requirements to be met, the hardware components available, developers experience and preferences, etc. Financial considerations often limit the realization of simple qualitative engineering solutions, such as a more powerful laser transmitter, a larger aperture of the receiving telescope and/or more sensitive detectors, which obviously lead to a greater range and better measurement and retrieval accuracy etc. To conduct quantitative trade-off studies, a significant number of instrumental parameters and external environmental factors must be taken into account $[1,2,2, \underline{4}, \underline{6}, \underline{12}]$. But it often 
remains indistinct, how much each subsystem and/or environmental parameter can quantitatively affect the ultimate performance.

The lidar systems analysis is traditionally based on examination of the signal-to-noise ratio (SNR) at the photodetector output $[1,24,6,7,9,12]$ as the most commonly used integrated efficiency criterion, which is a very useful tool for a lidar evaluation. But integrity is also a weak point because it obscures the impact of different components, which is desirable to be known for a system developer or user. If an influence of different factors is not evaluated separately, often it is difficult to assess the specific subsystem or capabilities of a measuring system taken all round $[13,14]$. In the absence of a generalized approach, the system designers use rather complex analytical expressions and empirical formulas often applicable only to a narrow range of parameters and special experimental setups $[6,10,15,16]$.

Earlier we performed a series of works $[\underline{1}, \underline{2}, \underline{13}-16]$ to introduce a dimensionless parameterization methodology and to describe and assess the lidar system performance from different points of view. They have shown that the universal parameterization applicable to many lidar technologies was useful as a tool for evaluation and design.

On the other hand, the experience has shown that a way to define some of five previously entered parameters in the dimensionless parametric lidar model $[15,16]$ is not always the most efficient because it is based on normalizing of certain lidar parameters to the receiver's threshold power determined in assumption of the sky background absence. As a result, parameters of such a model, which otherwise is useful and provides us with a preliminary assessment of the lidar system efficiency, do not provide a realistic estimation of a particular lidar potential and cannot compare different lidar systems during the daytime, when a majority of real systems are operated and when usually there is an intense sky background. In addition, the earlier model did not take the specifics of multiwavelength lidars into account.

The purpose of this paper is to stimulate further development for the earlier introduced methodology of lidar parameterization, to show real capabilities of a photon-counting mode applied to atmospheric lidar with SiPM detectors as an important addition to an analog mode detection and to show possibilities for wider application of the updated methodology adapted to real environmental conditions and extended to multiwavelength lidars.

\section{Modification of Dimensionless Parametric Model of Lidar}

The SNR at the lidar photodetector input $\psi_{X}$ can be decomposed as a product of five independent dimensionless parameters, each from different sources [16]:

$$
\psi_{X}=V Q_{X} W^{2} U^{-1} r^{-2},
$$

where $V$ is for ratio of the echo-signal power $P_{s 0}$ received from the reference range $R_{\text {ref }}$ of the reference molecular atmosphere to the photo-detector threshold power $P_{t 0}$ in the absence of background noise; $Q_{X}$ is for backscatter efficiency of the arbitrary species to the molecular reference; $W$ is for normalized atmospheric component determined by transparencies ratio of the current atmosphere state and the standard molecular atmosphere; $U$ is for ratio of photodetector's threshold powers taken in presence and in absence of background radiation; $r$ is for normalized range factor.

We consider the formerly entered $V$ parameter [16] as a fundamental characteristic of a lidar system that combines most of its important parameters and determines the potential of lidar in the most general form. In this regard, it seems appropriate to extend the practical use of the $V$ parameter by expanding it to all sounding wavelengths of multiwavelength lidars and adapting it to a reference level of daytime sky background, when using for threshold power estimates a more general approach than before.

\section{A. Introduction of Reference Background Radiance Used in Modified Models}

In support of the previous approach, we introduce a reference value of the sky background radiance in a typical and usual range of radiance, assuming that its value is quite appropriate to the extent of protection for a given lidar system against background of moderate radiance. Then we can reasonably predict a degree of deterioration of the lidar detection threshold under an exposure of background clutter of arbitrary radiance.

The continuous spectrum of solar radiation has a maximum intensity at the wavelength range of 430-500 $\mathrm{nm}$. In visible and infrared regions, the sun spectrum is close to the emission one of a black body with a temperature $T=6000 \mathrm{~K}$, and its radiance can be represented as $[\underline{10}, \underline{17}]$

$$
B_{\lambda}=2 \cdot h \cdot c^{2} \cdot \lambda^{-5}\left(e^{h c / \lambda k T}-1\right)^{-1}\left[\mathrm{~W} / \mathrm{m}^{2} \cdot \mathrm{sr} \cdot \mathrm{m}\right] .
$$

Since the peak of spectrum is located at the wavelength of $\lambda_{\max }=0.002898 / \mathrm{T}$ [17], the relative radiance distribution of solar radiation spectrum related to the chosen wavelength $\lambda$ can be deduced from the relation:

$$
b_{\lambda} \equiv B_{\lambda} / \mathrm{B}_{\lambda \max }=\left(\lambda_{\max } / \lambda\right)^{5} \cdot\left(\mathrm{e}^{h c / \lambda \max k T}-1\right) /\left(\mathrm{e}^{h c / \lambda k T}-1\right) .
$$

About $47 \%$ of the solar radiation on the Earth boundary layer reaches the Earth's surface. We assume the distribution of the daytime sky background by wavelengths corresponding to the solar radiation spectrum.

Typical range of the daytime sky background radiance at midlatitudes in the wavelength range of $0.4-0.7 \mu \mathrm{m}$ is as follows: $B_{\lambda}=10^{6} \ldots 3 \cdot 10^{8} \mathrm{~W}$. $\mathrm{m}^{-2} \mathrm{~m}^{-1} \mathrm{sr}^{-1}[10,17]$. For definiteness, we can take 
10 or more times less than maximum background radiance at $\lambda_{i}$ as reference values of daytime sky background radiance corresponding to different wavelengths $\lambda_{i}$ of laser sensing. For example, for $\lambda_{i}=532 \mathrm{~nm}$ the following value $B_{\text {ref } 532}=$ $10^{7} \mathrm{~W} \mathrm{~m}^{-2} \mathrm{~m}^{-1} \mathrm{sr}^{-1}$ can be used, which is about 30 times smaller than the maximum background intensity at midlatitudes and so forth.

The power of sky background received by lidar at real conditions is usually presented in the form:

$$
P_{b}(\lambda)=B_{\lambda} \cdot A_{r} \cdot \Omega \cdot \Delta \lambda(\lambda) \cdot \xi(\lambda) .
$$

Correspondingly, the value of sky background's reference power $P_{b \text { ref }}\left(\lambda_{i}\right)$ coming to a photodetector at the sounding wavelength $\lambda_{i}$ to be used in future assessments is determined only by the value of reference radiance $B_{\text {ref }}\left(\lambda_{i}\right)$ and by parameters of lidar receiving system $[\underline{12}, \underline{17]}$ :

$$
P_{\text {bref }}\left(\lambda_{i}\right)=B_{\text {ref }}\left(\lambda_{i}\right) \cdot A_{r} \cdot \Omega \cdot \Delta \lambda\left(\lambda_{i}\right) \cdot \xi\left(\lambda_{i}\right) .
$$

\section{B. Adaptation of $V$ Parameter to Reference Background Radiance and Multiwavelength Lidar}

In this subsection, we will adapt the $V$ parameter as an input SNR for the reference range and atmosphere to the reference background radiance and extend its application to multiwavelength lidars.

The lidar's output SNR can be presented in a simple form [ㅁ, $\underline{9}]$ as follows:

$$
\rho_{\text {out }}^{2}=P_{s}^{2} /\left[P_{q}\left(P_{s}+P_{b}\right)+P_{n}^{2}\right],
$$

where $P_{s}$ is the received lidar signal power. In the quantum noise limit of the receiver sensitivity that often takes place in lidar systems, the quantum noise power $P_{q}$, which depends on the factor of excess noise $F$, the transmission band $\Delta f$, the wavelength $\lambda$, the quantum efficiency $\eta$, and provides a useful scale to judge the power levels of signal, background, and receiver's internal noise.

As is resulted from quadratic Eq. (3), at daytime measurements, when the absolute threshold signal power $P_{t}$ (or power of minimal detectable signal, $\left.P_{\min S}\right)$ is determined by the

$$
P_{s} / P_{q}=\frac{1}{2} \rho_{\text {out }}^{2} /\left[1+\sqrt{1+4\left(P_{b} / P_{q}+P_{n}^{2} / P_{q}^{2}\right) / \rho_{\text {out }}^{2}}\right]
$$

sky background, it can be written as follows under conditions of the reference background $B_{\text {ref }}\left(\lambda_{i}\right)$ :

$$
\begin{aligned}
& P_{t B \text { ref }}\left(\lambda_{i}\right) \\
& \equiv P_{t}\left(\lambda_{i}, B_{\text {ref }}\right)=1 / 2 \rho_{\text {out }}^{2} P_{q}\left(\lambda_{i}\right) \\
& \quad\left\{1+\sqrt{\left.1+\left(4 / \rho_{\text {out }}^{2}\right)\left[P_{b r e f}\left(\lambda_{i}\right) / P_{q}\left(\lambda_{i}\right)+P_{n}^{2}\left(\lambda_{i}\right) / P_{q}^{2}\left(\lambda_{i}\right)\right]\right\}} .\right.
\end{aligned}
$$

When analyzing the potential of any lidar system and trying to specify the particular lidar system response at standard atmospheric conditions, in $[13,16]$ it was proposed to adapt the optical parameters of sounded medium to parameters of the molecular atmosphere with pre-agreed parameters. This allows us to predict the behavior of lidar at certain atmospheric conditions more effectively.

As noted above, the previously entered reference medium $[13,16]$ enabled adapting of the arbitrary optical "weather" to standardized parameters of molecular atmosphere. The modified universal lidar parameter for each sounding wavelength can be obtained by using the following three factors: (a) introduction of standardized conditions of external environment's background radiation in the form of its brightness according to Eq. (2); (b) taking into account the background brightness in Eq. (4) for threshold power of lidar signal; and (c) subsequent joint account of reference environment and reference background brightness in analytical expressions for $V$ parameter calculations.

So when comparing different types of lidars, it is useful to identify the $V$ parameter as a universal dimensionless constant of a multiwavelength system at specific wavelength $\lambda=\lambda_{i}$ (i.e., $V_{i}$ parameter at $\lambda_{i}$ ) that characterizes the lidar's capability for remote sensing and describes not only its energetic potential, but the lidar instrument's immunity to background radiation as well. Numerically, the $V$ parameter can be redefined as a ratio of the echo-signal power $P_{s 0 i}$ received from the reference range $R_{\text {ref }}$ of the reference atmosphere (for example, at molecular atmosphere conditions) to the photodetector's threshold power $P_{t B r e f}\left(\lambda_{i}\right)$ at reference background conditions (for example, at conditions of «averaged» sky background brightness in the middle latitudes):

$$
V_{i} \equiv P_{s 0}\left(\lambda_{i}\right) / P_{t B r e f}\left(\lambda_{i}\right) .
$$

In Fig. 1, a schematic illustration of the $V$ parameter formation principle, which includes a joint account of the reference environment and the reference sky background.

Let's write the $V$ parameter in detail, taking into account the lidar system parameters by adapting the Eq. ( $\underline{4})$ to the reference background density $B_{\text {ref }}$ :

$$
V\left(\lambda_{i}\right) \equiv \frac{P_{s 0}\left(\lambda_{i}\right)}{P_{t B \mathrm{ref}}\left(\lambda_{i}\right)}=\frac{P_{s 0}\left(\lambda_{i}\right)}{\frac{1}{2} \rho_{\text {out }}^{2} P_{q}\left(\lambda_{i}\right)\left[1+\sqrt{1+\left(4 / \rho_{\text {out }}^{2}\right)\left[P_{b \mathrm{ref}}\left(\lambda_{i}\right) / P_{q}\left(\lambda_{i}\right)+P_{n}^{2}\left(\lambda_{i}\right) / P_{q}^{2}\left(\lambda_{i}\right)\right]}\right]},
$$




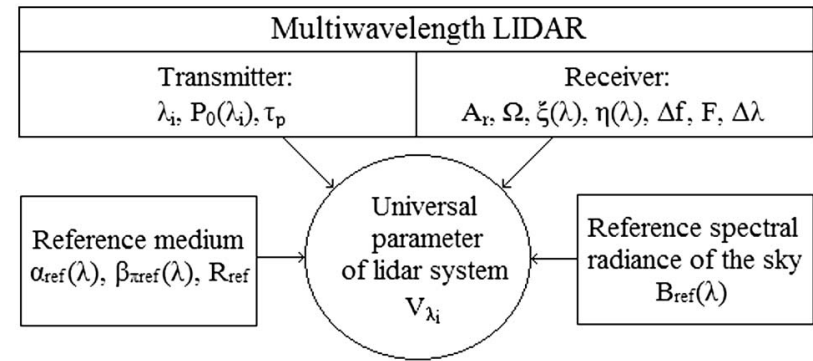

Fig. 1. Schematic illustration of the universal $V$ parameter formation principle.

where $\quad P_{s 0}\left(\lambda_{i}\right)=1 / 2 c \tau_{p} P_{0}\left(\lambda_{i}\right) A_{r} \xi\left(\lambda_{i}\right) \beta_{\pi \mathrm{ref}}\left(\lambda_{i}\right) T_{0}^{2}$ $\left(\lambda_{i}, \alpha_{\text {ref }}, R_{\text {ref }}\right) R_{\text {ref }}^{-2} P_{b \text { ref }}\left(\lambda_{\mathbf{i}}\right)=B_{\text {ref }}\left(\lambda_{\mathbf{i}}\right) \cdot A_{r} \cdot \Omega \cdot \Delta \lambda\left(\lambda_{\mathbf{i}}\right) \cdot \xi\left(\lambda_{\mathbf{i}}\right)$, and $\rho_{\text {out }}$ is the output SNR.

Now we can write out in detail three important cases, which are to be met with in practice with different photodetectors (i.e., with different $P_{q}$ ), when the $P_{b r e f}$ value is chosen, to simplify the formula in Eq. (5), by selecting the components characterizing the lidar system parameters and atmospheric parameters, and using for threshold conditions $\rho_{\text {out }}=1$ in its final form:

(a) $P_{\text {bref }} \gg P_{q}$ :

$$
\begin{aligned}
V\left(\lambda_{i}\right)= & \tau_{p} P_{0}\left(\lambda_{i}\right) \sqrt{\frac{c A_{r} \xi\left(\lambda_{i}\right) \lambda_{i} \eta\left(\lambda_{i}\right)}{8 h F \Delta f B_{\mathrm{ref} \lambda i} \Omega \Delta \lambda}} \\
& \times \beta_{\pi \mathrm{ref}}\left(\lambda_{i}\right) T_{0}^{2}\left(\lambda_{i}, \alpha_{\mathrm{ref}}, R_{\mathrm{ref}}\right) R_{\mathrm{ref}}^{-2},
\end{aligned}
$$

(b) $P_{\text {breff }} \approx P_{q}$ :

$$
\begin{aligned}
V\left(\lambda_{i}\right)= & \frac{\tau_{p} P_{0}\left(\lambda_{i}\right) A_{r} \xi\left(\lambda_{i}\right) \lambda_{i} \eta\left(\lambda_{i}\right)}{2(1+\sqrt{5}) h F \Delta f} \\
& \times \beta_{\pi \mathrm{ref}}\left(\lambda_{i}\right) T_{0}^{2}\left(\lambda_{i}, \alpha_{\mathrm{ref}}, R_{\mathrm{ref}}\right) R_{\mathrm{ref}}^{-2},
\end{aligned}
$$

(c) $P_{\text {bref }} \ll P_{q}$ :

$$
\begin{aligned}
V\left(\lambda_{i}\right)= & \frac{\tau_{p} P_{0}\left(\lambda_{i}\right) A_{r} \xi\left(\lambda_{i}\right) \lambda_{i} \eta\left(\lambda_{i}\right)}{4 h F \Delta f} \\
& \times \beta_{\pi \mathrm{ref}}\left(\lambda_{i}\right) T_{0}^{2}\left(\lambda_{i}, \alpha_{\mathrm{ref}}, R_{\mathrm{ref}}\right) R_{\mathrm{ref}}^{-2} .
\end{aligned}
$$

The physical interpretation of $V\left(\lambda_{i}\right)$ parameter is a ratio of lidar signal power at sounding the standardized molecular atmosphere with reference parameters to the threshold power of the same lidar at standardized/reference exposure conditions of daytime sky background.

The convenience of the redefined dimensionless parameter $V\left(\lambda_{i}\right)$ is that it uniquely determines the power potential of lidar; it takes into consideration all important parameters of its transmitting and receiving channels and quantifies its ability to sound the medium at a wavelength $\lambda_{i}$ and a distance $R_{\text {ref }}$ on and example of the standard molecular atmosphere as well as the lidar's ability to withstand the effects of external background with a reference spectral density $B_{\text {ref }}$, since the sky background almost always exists at daytime operation. This universal $V\left(\lambda_{i}\right)$ parameter makes it easy to compare the potential of a multiwavelength lidar at each operating wavelength to different lidars and predict their response, when sounding different media and under the influence of different background radiation. Practical use of the $V$ parameter allows us to better considering of specifics of engineering implementation in different and independently designed lidar systems for evaluation of relative effectiveness of any given engineering solutions within the same multiwavelength lidar system.

As Eqs. (4)-(7) show, to estimate the $V$ parameter value for existing or projected lidar instruments, one only needs to know the parameters of transmitting and receiving subsystems, the optical parameters of a standard molecular atmosphere and of a reference sky background. According to Eqs. (무)-( $\underline{8})$ and due to wide variations of lidar instrument parameters for different lidar types $\left[\lambda_{i}, \tau_{p}, P_{0}\left(\lambda_{i}\right), R_{\text {ref }}, A_{r}\right.$, $\left.\xi\left(\lambda_{i}\right), \Omega, \Delta \lambda\left(\lambda_{i}\right), \Delta f, \mathrm{NEP}\right]$, the value of the $V$ parameter can change dramatically from $10^{-11}$ to $10^{7}$. It is easy to see that large values of $V$ lead to improvement of lidar characteristics, but, in turn, these values correspond to more expensive electro-optical components.

This is precisely why the so-defined $V$ parameter identifies a particular lidar implemented in specific engineering solutions and characterizes their performance and efficiency.

\section{Modification of the Lidar's $U$ Parameter as an Extent of the Detection Threshold Degradation under Intense Background}

As a matter of record provided in our paper [15], and instead of a generalized dimensionless $U$ parameter of the system introduced earlier in [16] as a ratio of photodetector's threshold powers $P_{t 0}$ and $P_{t}$ taken in presence/absence of background radiation, we will use a set of parameters characterizing the lidar receiving system and degree of its immunity to background radiation from the environment, which is distributed by wavelength of laser sensing. On the basis of the reference value of the daytime sky's background brightness used in Eq. (2), we will redefine the set of $U$ parameters and represent it as a ratio of photodetector's sensitivity thresholds $P_{t B}$ and $P_{t B \text { ref }}$, when $P_{t B}$ is established with background radiation of arbitrary radiance available, and $P_{t B \text { ref }}$ is determined by reference noise caused by the cloudy sky background with spectral density $B_{\text {ref. }}$ In both the cases, the wavelength distribution of brightness is considered relevant to the spectrum of solar radiation:

$$
\begin{aligned}
U_{i} \equiv & P_{t}^{B}\left(\lambda_{i}\right) / P_{t}^{B \operatorname{ref}}\left(\lambda_{i}\right) \\
= & {\left[1+\sqrt{1+\frac{4}{\rho_{\text {out }}^{2}}\left(\frac{P_{b}\left(\lambda_{i}\right)}{P_{q}\left(\lambda_{i}\right)}+\frac{P_{n}^{2}\left(\lambda_{i}\right)}{P_{q}^{2}\left(\lambda_{i}\right)}\right)}\right] } \\
& /\left[1+\sqrt{1+\frac{4}{\rho_{\text {out }}^{2}}\left(\frac{P_{b \mathrm{ref}}\left(\lambda_{i}\right)}{P_{q}\left(\lambda_{i}\right)}+\frac{P_{n}^{2}\left(\lambda_{i}\right)}{P_{q}^{2}\left(\lambda_{i}\right)}\right)}\right] .
\end{aligned}
$$


The physical meaning of the newly introduced $U$ parameter is an extent of the lidar photodetector's detection threshold degradation under intense daytime sky background (Fig. 2).

Since it is true for SiPM and PMT detectors: $P_{n} \ll$ $P_{q}$ (although for SiPMs the inequality is much stronger), the formula in Eq. (9) can be simplified. Then it is easy to see that under intense daytime sky background with $P_{b} \gg P_{q} \gg P_{n}$, the $U$ parameter being a degree of detection threshold, which increases under the intense background radiation, for $\rho_{\text {out }}=1$ becomes as follows:

$$
U_{i}=\frac{2 \sqrt{B_{\lambda i} A_{r} \xi\left(\lambda_{i}\right) \Omega \Delta \lambda \lambda_{i} \eta\left(\lambda_{i}\right) / h c F \Delta f}}{1+\sqrt{1+2 B_{\mathrm{ref} \lambda i} A_{r} \xi\left(\lambda_{i}\right) \Omega \Delta \lambda \lambda_{i} \eta\left(\lambda_{i}\right) / h c F \Delta f}},
$$

where $B_{\lambda i}$ is the spectral radiance of the sky zone falling in the receiver field of view at $\lambda=\lambda_{i}$.

Once we have chosen the reference background brightness to provide the reference background power to be of the same order of magnitude as the photodetector's quantum noise, i.e., $P_{b \text { ref }} \sim P_{q}$, then Eq. (10) can be rewritten in a simpler form as follows:

$$
U_{i} \approx \sqrt{P_{b}\left(\lambda_{i}\right) / P_{q}\left(\lambda_{i}\right)}=\sqrt{B_{\lambda i} A_{r} \xi\left(\lambda_{i}\right) \Omega \Delta \lambda \lambda_{i} \eta\left(\lambda_{i}\right) / h c F \Delta f} .
$$

If lidar operates at night time, then $P_{q} \gg P_{n}$ and $P_{q} \gg P_{b}$, and the following is deduced from Eq. (9):

$$
\begin{aligned}
U_{i} & =\frac{2}{1+\sqrt{1+\frac{4}{\rho_{\text {out }}^{2}} \frac{P_{\text {bref }}\left(\lambda_{i}\right)}{P_{q}\left(\lambda_{i}\right)}}} \\
& =\frac{2}{1+\sqrt{1+2 B_{\mathrm{ref} \lambda i} A_{r} \xi\left(\lambda_{i}\right) \Omega \Delta \lambda \lambda_{i} \eta\left(\lambda_{i}\right) / h c F \Delta f}} .
\end{aligned}
$$

\section{Experimental Studies with SiPMs as Photodetectors for Laser Remote Sensing}

Within the last few years, research groups in many countries exhibit activity in development of SiPM detectors, which allow us to expand traditional application of high-sensitivity photodetectors on PMTs and avalanche photodiodes and demonstrate

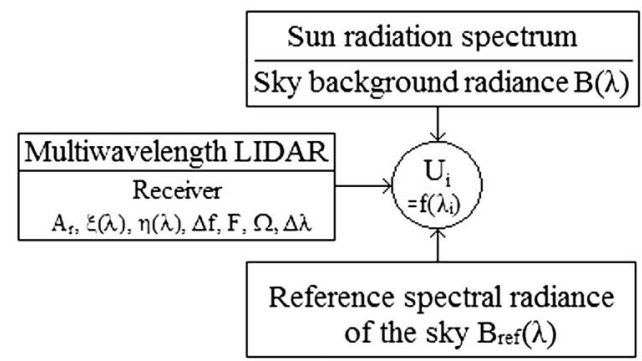

Fig. 2. Schematic illustration of the $U$ parameter formation principle. new properties that were unattainable before $[18,19]$. At the same time, we know the only published paper [20] dedicated to SiPMs in analog mode and used in atmospheric lidars, although it is clear that prospects for their application in lidars are much wider.

\section{A. Experimental Setup and Raw Signal Examples}

Since up to now the SiPM photodetectors are not commonly used in lidars, we have conducted fullscale lidar experiments with SiPM photodetectors in both analog and photon counting modes. In parallel, we measured the echo-signals with traditional PMT detectors. Experiments were performed in a specialized laboratory at UPC, Barcelona (Spain), on the basis of a six-channel lidar automated measurement system, which is used in the EARLINET, the European Aerosol Research Lidar Network. During many tests, a dual-channel polychromator was used, and one of its channels had the highly sensitive photodetectors SiPM-Hamamatsu S1036233-100C (hereinafter SiPM-H) and SensL 30035 (hereinafter SiPM-S) built in. To compare features of two SiPM detectors, in the second channel of polychromator, we have used PMT Hamamatsu R7400P (hereinafter PMT-H), which is widely used today in atmospheric lidars.

A flowchart of the experiment is provided in Fig. 3. Its specificity lies in the fact that, according to the authors' best knowledge, the use of SiPM photodetectors in photon-counting mode for atmospheric lidar applications was not described in literature before.

\section{B. Specific Features of SiPM and PMT Detectors Characteristic Parameters}

Specific features and differences between commonly used PMTs and relatively new silicon photomultipliers SiPMs, which should be taken into account are given in Table 1, where a number of main parameters of silicon photomultipliers under consideration, namely, SiPM SensL 30035 and SiPM Hamamatsu

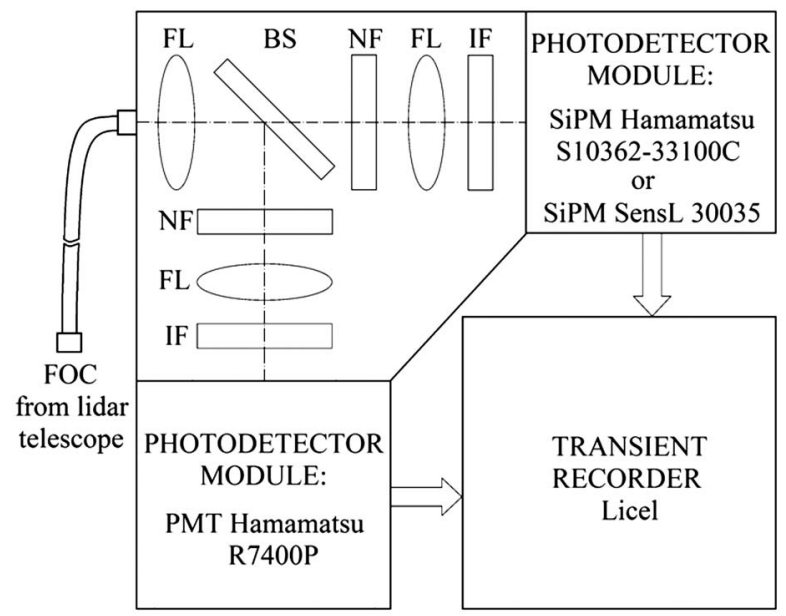

Fig. 3. Flow chart of experimental setup. Designations: FOC, fiber-optical cable; FL, focusing lens; BS, beam splitter; NF, neutral filter; IF, interference filter. 
Table 1. Some Parameters of SiPMs and PMT Used

\begin{tabular}{|c|c|c|c|c|c|c|c|c|}
\hline Photodetector & $\begin{array}{c}\text { Internal } \\
\text { Gain M }\end{array}$ & $\begin{array}{c}\text { Dark Current } \\
\quad I_{d A}, A\end{array}$ & $\begin{array}{c}\text { Excess Noise } \\
\text { Factor } F\end{array}$ & Active Area & $\begin{array}{l}\text { Microcell } \\
\text { Size }\end{array}$ & $\begin{array}{l}\text { Number of } \\
\text { Microcells }\end{array}$ & $\begin{array}{l}\text { Operating } \\
\text { Voltage } V_{\text {op }}\end{array}$ & $\begin{array}{c}\text { Peak } \\
\text { Wavelength } \lambda_{p}\end{array}$ \\
\hline SiPM-S: SensL 30035 & $2.4 \times 10^{6}$ & $3.0 \times 10^{-6}$ & 1.30 & $3 \mathrm{~mm} \times 3 \mathrm{~mm}$ & $35 \mu \mathrm{m}$ & 4774 & $29.5 \mathrm{~V}$ & $500 \mathrm{~nm}$ \\
\hline $\begin{array}{l}\text { SiPM-H: Hamamatsu } \\
\text { S10362-33-100C }\end{array}$ & $2.4 \times 10^{6}$ & $3.0 \times 10^{-6}$ & 1.42 & $3 \mathrm{~mm} \times 3 \mathrm{~mm}$ & $100 \mu \mathrm{m}$ & 900 & $70 \pm 10 \mathrm{~V}$ & $440 \mathrm{~nm}$ \\
\hline $\begin{array}{l}\text { PMT-H: Hamamatsu } \\
\text { R7400P }\end{array}$ & $10^{6}$ & $2.0 \times 10^{-10}$ & 1.20 & $\varnothing 8 \mathrm{~mm}$ & - & 一 & $800 \mathrm{~V}$ & $420 \mathrm{~nm}$ \\
\hline
\end{tabular}

S10362-33-100C are presented, together with parameters of PMT Hamamatsu R7400P, which is often used in atmospheric lidars and therefore is taken for comparison.

Spectral dependences of the quantum noise $P_{q}$ and internal noise $P_{n}$ powers of photodetectors under discussion and their photon detection efficiency curves $\eta(\lambda)$ are displayed in Fig. 4. Similar dependences for the PMT general introduced earlier in [Section 4.1 in Ref. 15] given for comparison, where the following values of PMT parameters have been used in estimations: $\quad F_{\text {PMT-general }}=1.3 ; \quad M_{\text {PMT-general }}=10^{6}$; $I_{d \text { PMT-general }}=3 \times 10^{-10} \mathrm{~A}$.

These calculations will be used later for generalized estimations.

\section{Primary Processing of Measurement Results}

In Fig. 5, we present a few examples selected from a database of lidar echo-signal measurements with SiPM and PMT photodetectors in analog and photon-counting modes. In the upper part of Fig. 5, the range-square compensated echo-signals $\left(P \cdot R^{2}\right)$ detected at $\lambda=532 \mathrm{~nm}$ by the SiPM-H (analog mode and photon counting) and PMT-H (photon counting) are shown. In the lower part of Fig. 5 , one can find the echo-signals detected by the SiPM-S (analog mode and photon counting) and PMT-H (analog mode).

As is seen from above, the results of lidar measurements and primary processing of signals performed by use of SiPM-S and SiPM-H detectors are in excellent agreement with those obtained by traditionally used PMTs.

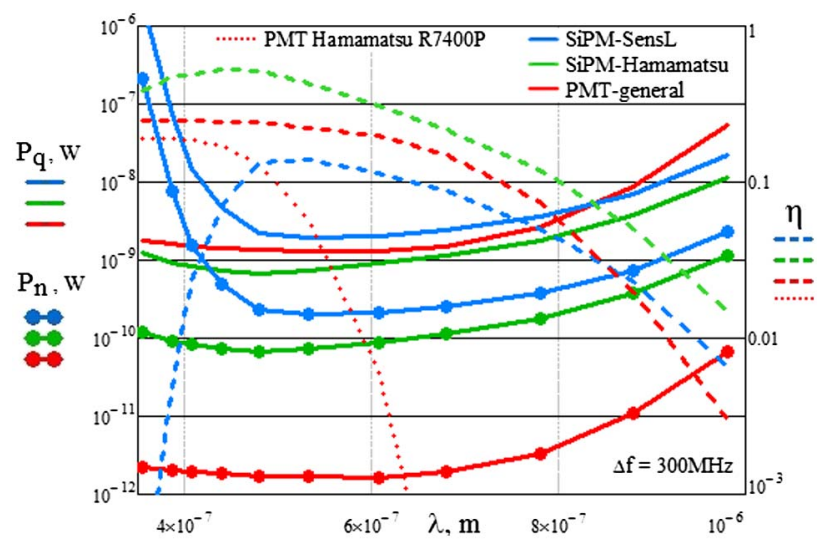

Fig. 4. Quantum noise $P_{q}$, internal noise $P_{n}$ of photodetectors, and their quantum efficiency $\eta$ as a function of wavelength $\lambda$.
Some difference in SiPM and PMT signal shapes can be easily explained by some inequality of optical signal shapes entering the photodetectors inputs after the beam splitter (BS).

In Fig. 6 , the results of primary processing of the automate $\bar{d}$ lidar measurements at $\lambda=532 \mathrm{~nm}$ with the using of SiPM-S in the first polychromator channel of the UPC lidar, and of PMT-H in its second channel are shown. Figure 6 includes a color illustration of the optical weather changes in the form of a range-square-corrected lidar signal within $10 \mathrm{~min}$ measurement period; a range profile of backscattered coefficient inverted from the $6 \mathrm{~km}$ path lidar

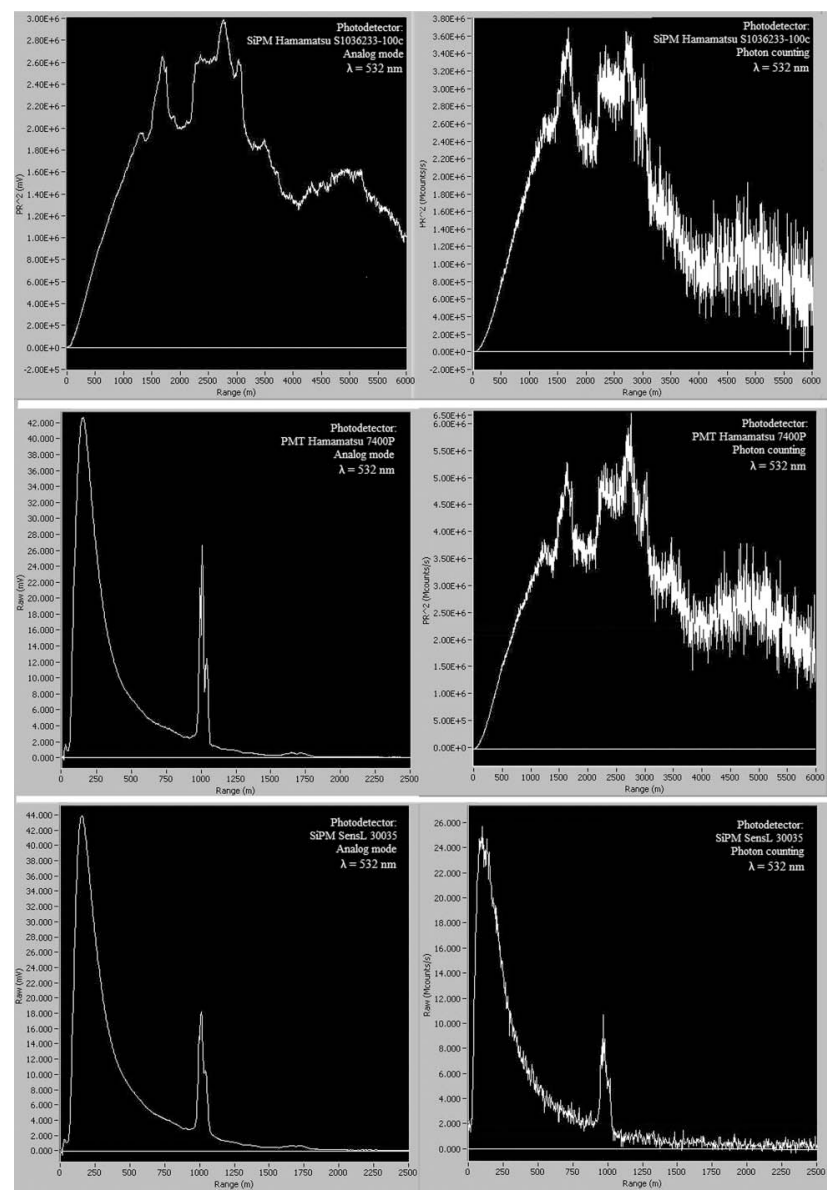

Fig. 5. Echo signals of lidar with SiPM and PMT photodetectors in different modes. Top: the range-square-compensated $\left(P \cdot R^{2}\right)$ SiPM Hamamatsu (analog mode and photon counting). Middle: the PMT-Hamamatsu (analog mode and photon counting). Bottom: SiPM SensL (analog mode and photon counting). 

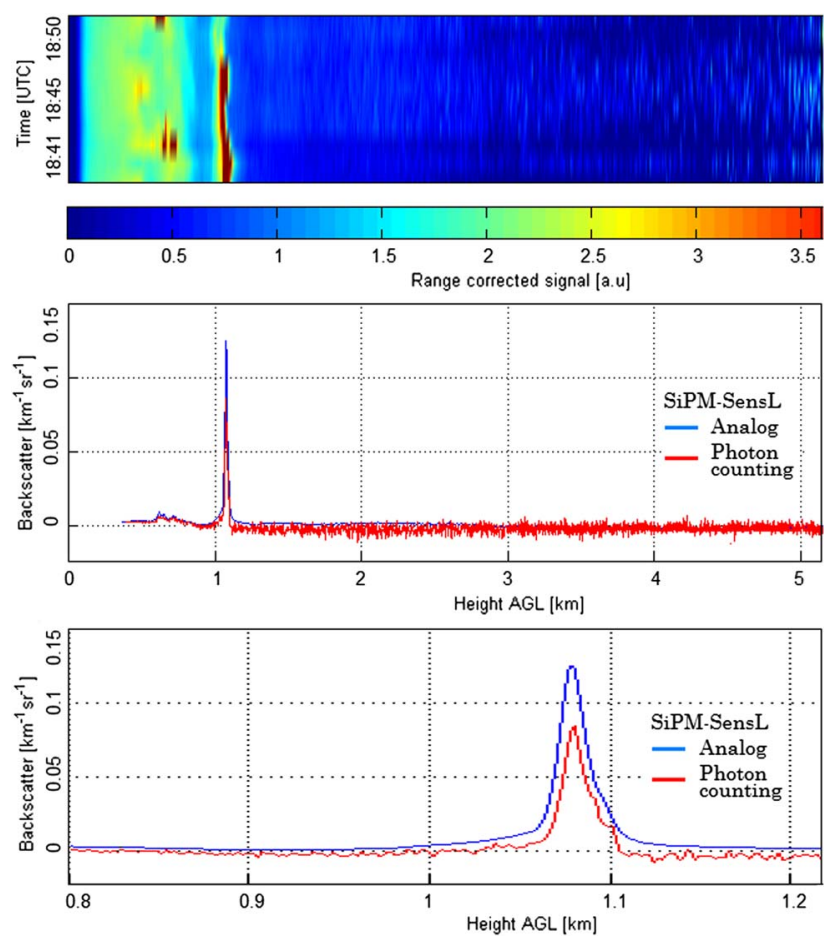

Fig. 6. Range-compensated lidar signals' time series (top) and backscattering coefficient inversions (middle and bottom) with SiPM detector SensL 30035 in analog (blue) and photon-counting (red) modes.

signal; and the same signal considered on $0.5-1.5 \mathrm{~km}$ interval.

The results of lidar measurements with photodetectors working in analog and photon-counting modes showed that SiPMs can be successfully used in lidar systems, and in all operating modes, both together with PMTs and instead of them. Lidar measurements conducted have shown in practice the actual feasibility of photon-counting mode implementation in atmospheric SiPM-lidar as an important addition to the analog mode detection [20], which significantly increases the lidar capabilities. In this case, the raw experimental data (here: «raw» = raw data + preprocessing) of lidar measurements can be subjected to all methods of traditional algorithmic processing of lidar echoes.

It is clear that specific characteristics of SiPMs, particularly their noise characteristics, gain's temperature dependences, and other parameters may require further researching and development. But it is quite sure that SiPMs present a very promising class of photodetectors for use in lidars, and they will be used in practice in the near future in wider scope.

\section{Application of Dimensionless Parameterization Model to Lidars with SiPMs in Comparison with PMTs}

When analyzing potential and capabilities of lidars with SiPM-detectors, not only spectral characteristics of photodetectors should be taken into account, but also the related parameters characterizing the system efficiency when measuring the lidar signals.
A. $U$ and $V U_{\text {equ }}$ Parameters of LIDAR with SiPMs in Comparison with PMTs

Coming back to the formulae in Eqs. (10) to (12), the curves $U=f\left(\lambda_{i}\right)$ for lidar with SiPM-S/SIPM-H/ PMT general detectors under the sky background brightness $B_{\lambda}=10^{8}$ and $10^{9} \mathrm{~W} \cdot \mathrm{m}^{-2} \cdot \mathrm{sr} \cdot \mathrm{m}^{-1}$ can be represented as shown in Fig. 7(a). Dependences $U=f\left(B_{\lambda}\right)$ for lidar operating with the same photodetectors at probing wavelengths of 355 and $532 \mathrm{~nm}$ are provided in Fig. 7(b).

For better illustration of how much the sky background of different brightness affects the ability of photodetectors (SiPM SensL, SiPM Hamamatsu, and PMT general) compared to remote sensing, we introduce a unified $V U_{\text {equ }}$ parameter. Taking into account that for SiPM- and PMT-based lidar photodetectors the quantum noise is stronger than the internal noise $\left(P_{q} \gg P_{n}\right.$, though for PMTs it is much stronger), a combined $V U_{\text {equ }}$ parameter can be written as
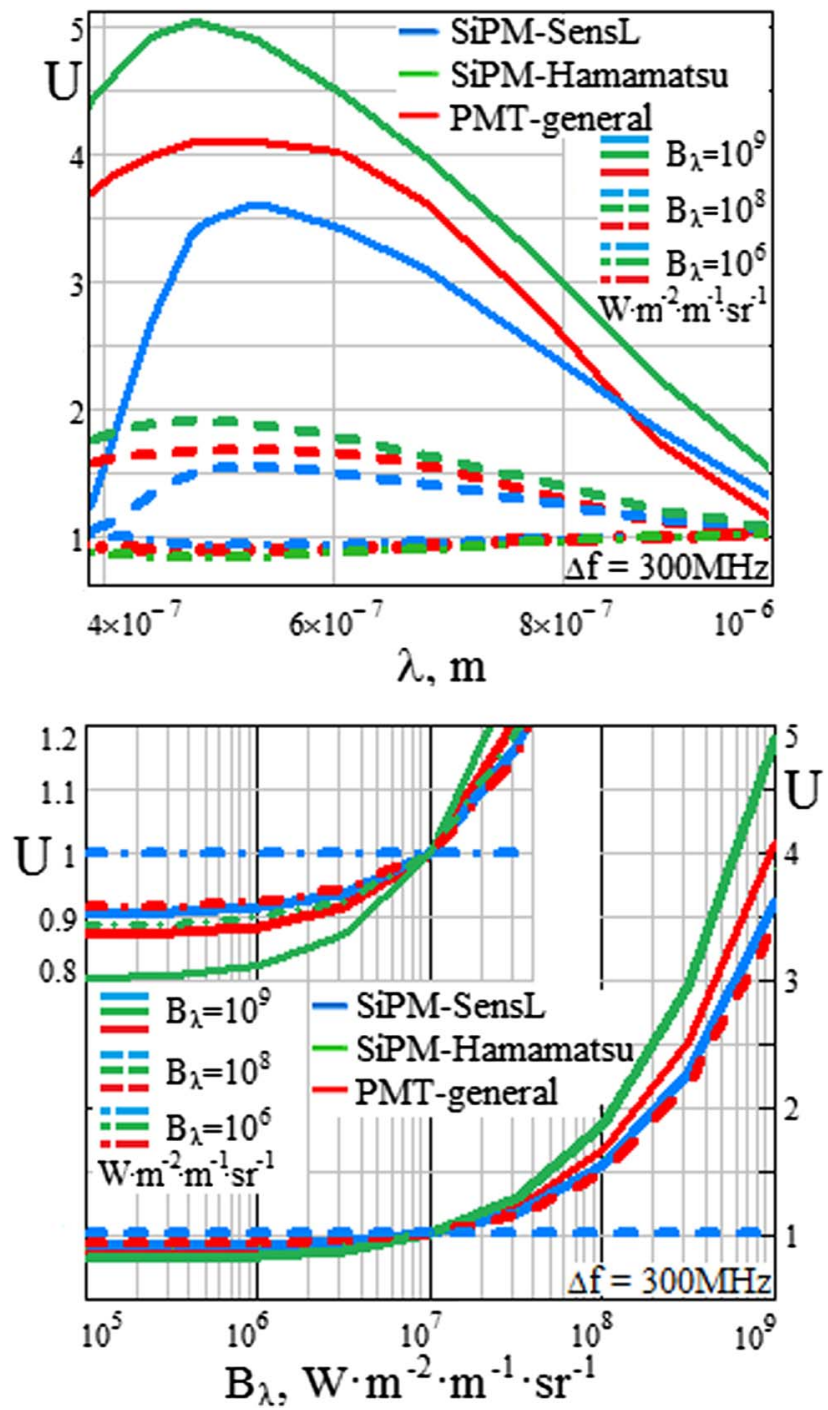

Fig. 7. $U$ parameter as a function of (a) wavelength and (b) of brightness of the sky background brightness. 


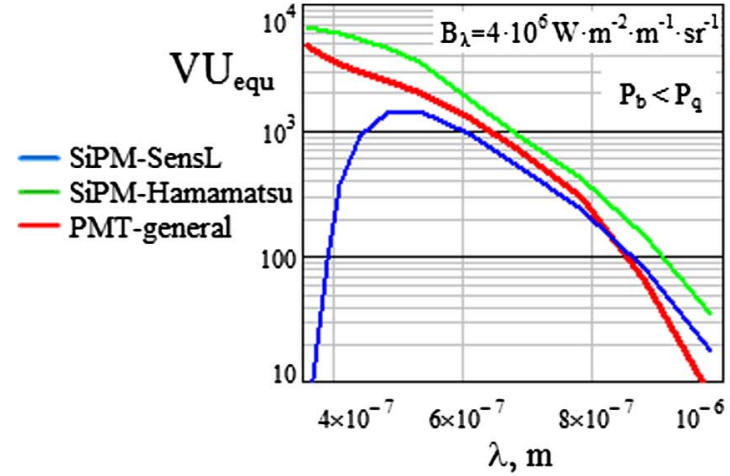

(a)



(b)

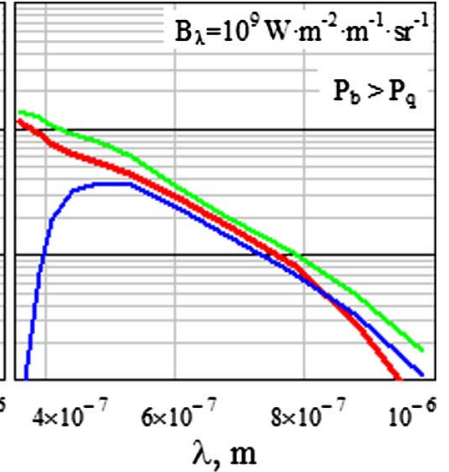

(c)

Fig. 8. United $V U_{\text {equ }}$ parameter as an illustration of effect of varying brightness of sky background effect on remote sensing abilities of compared photodetectors: SiPM SensL, SiPM Hamamatsu and PMT general: (a) weak sky background $\left(P_{b} \ll P_{q}\right)$; (b) moderate sky background $\left(P_{b} \approx P_{q}\right) ;(\mathrm{c})$ strong sky background $\left(P_{b} \gg P_{q}\right)$ with $B_{\lambda}=4 \cdot 10^{6} / 4 \cdot 10^{7} / 10^{9} \mathrm{~W} \cdot \mathrm{m}^{-2} \cdot \mathrm{sr} \cdot \mathrm{m}^{-1}$, respectively; $R=1 \mathrm{~km}$.

$$
\begin{aligned}
V U_{\text {equ }}\left(\lambda_{i}\right) & \equiv \frac{V\left(\lambda_{i}\right)}{U\left(\lambda_{i}\right)} \\
& =\frac{c \tau_{p} P_{0}\left(\lambda_{i}\right) A_{r} \xi\left(\lambda_{i}\right) \beta_{\pi}\left(\lambda_{i}, \alpha_{i}\right) T_{0}^{2}\left(\lambda_{i}, R_{0}\right) R_{0}^{-2}}{\rho_{\text {out }}^{2} P_{q}\left(\lambda_{i}\right)\left[1+\sqrt{1+\left(4 / \rho_{\text {out }}^{2}\right)\left[P_{b}\left(\lambda_{i}\right) / P_{q}\left(\lambda_{i}\right)\right]}\right]} .
\end{aligned}
$$

According to Eq. (13), the combined $V U_{\text {equ }}$ parameter under conditions of strong, moderate, and weak daytime sky background, when its power $P_{b} \gg P_{q}, P_{b} \approx P_{q}$ and $P_{b} \ll P_{q}$ at the sky brightness $B_{\lambda}=10^{9} / 4 \cdot 10^{7} / 4 \cdot 10^{6} \mathrm{~W} \cdot \mathrm{m}^{-2} \cdot \mathrm{sr} \cdot \mathrm{m}^{-1}$, respectively, is given in Fig. $\underline{8}$. It illustrates the $V U_{\text {equ }}$ parameter's behavior in near UV visible near IR ranges for SiPM and PMT detectors.

Curves are based on calculations by the analytical model Eq. (푸) for $P_{b} \ll P_{q}, P_{b} \approx P_{q}, P_{b} \gg P_{q}$.

\section{B. Estimation of Minimal Detectable Values of the $Q$} Parameter for SiPM Detectors in Comparison with PMTs

The effectiveness of medium backscatter is directly characterized by the value of $Q$ parameter, which simply describes the arbitrary species' backscatter efficiency to the molecular reference and was mentioned in Section 1 , so that calculating the minimum value of $Q$ for a given wavelength determines a minimum detectable target or a scattering medium. Sample calculation results of $Q_{x}$ values for different types of lidars and for different intervening atmospheres for a range of $Q_{x}$ parameter magnitudes are as follows [16]: for topographic lidar $Q_{x}=10^{2} \ldots 10^{4}$; for elastic lidar in aerosol atmosphere $Q_{x}=10^{0} \ldots 10^{2}$; for elastic lidar in molecular atmosphere $Q_{x}=10^{-1} \ldots 10^{1}$; for Raman lidar detecting, for example, $N_{2}$ and $\mathrm{H}_{2} \mathrm{O}$ it will be $Q_{x}=10^{-5} \ldots 10^{-3}$ and $Q_{x}=10^{-7} \ldots 10^{-5}$, correspondingly.

To determine the lower limit of lidar detection, let's equate the SNR in Eq. (1) to one, which leads to the following expression: $V \cdot Q_{X} \cdot W^{2} \cdot U^{-1}$. $r^{-2}=1$. In Fig. $\underline{9}$, dependences of normalized parameter $Q_{X \text { norm }}$ as a function of wavelength for various photodetectors (SiPMs from SensL and Hamamatsu and general PMTs) under the daylight sky background effects displayed in the form of bands within the boundaries defined by strong background/lack of it are shown.

On the basis of these calculations, one can estimate, for example, the concentration of gases/vapors measurable by the Raman lidar using any of the three photodetectors. Taking into account an appropriate of backscattering cross section of the scattering medium and the reference molecular atmosphere, it is possible to estimate the lower limit of the $Q_{x}$ parameter, which allows us to calculate the minimum detectable concentration of gas or vapor.

\section{Reduction of Operating Range Under Intense} Background for SiPMs in Comparison with PMTs

From Eq. (1) it is easy to determine the normalized operation range of lidar $r=R_{\max } / R_{\text {ref }}$ by assuming the input $\mathrm{S} / \mathrm{N}$ ratio $\psi_{X}=1$. For a simple case of sensing the molecular atmosphere at $Q_{x}=1$ it was obtained in [16]

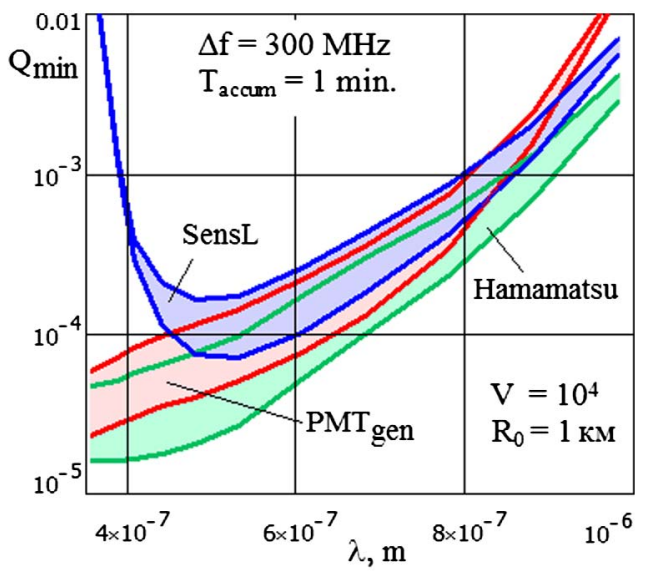

Fig. 9. Minimum detectable target or scattering medium characterized by bands of minimum achievable values of $Q_{X \text { norm }}$ parameter as a function of wavelength within the boundaries corresponding to strong daylight sky background and lack of background for photodetectors SiMP SensL, SiMP Hamamatsu, PMT general. $R_{0}=1 \mathrm{~km} ; T_{\text {accum }}=1 \mathrm{~min} ; \Delta f=3 \cdot 10^{8} \mathrm{~Hz}$. 

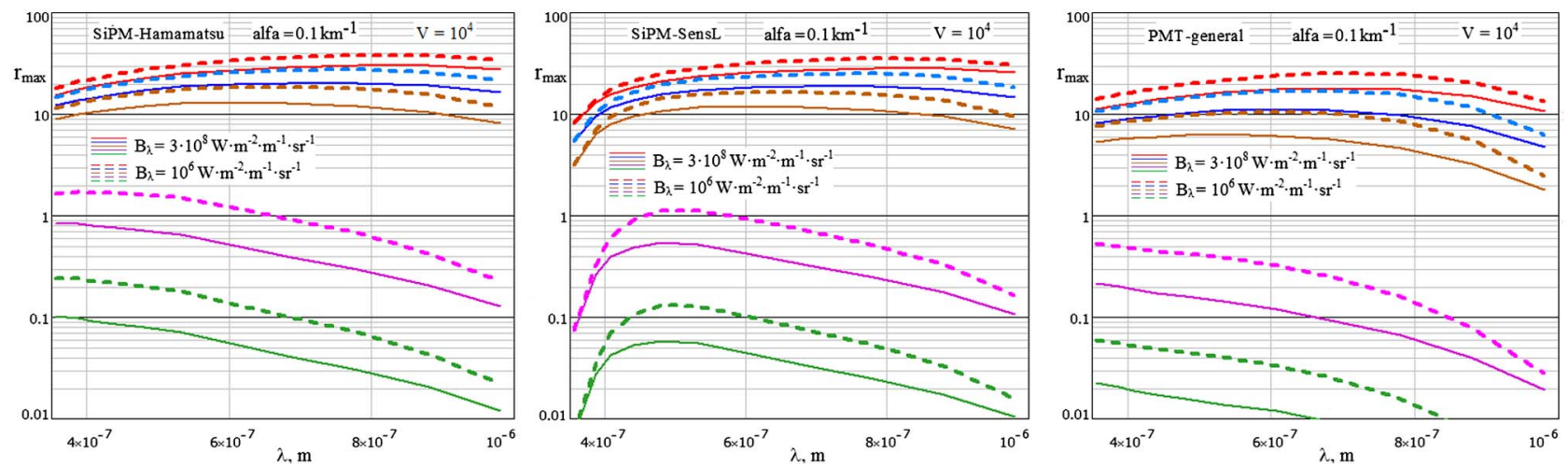

Fig. 10. Relative maximum operation range of the SiMP Ha, SiMP Se, PMT gen as a function of wavelength $r_{\max }=f(\lambda)$ for different $B_{\lambda}$ and $Q: B_{\lambda}=10^{6} \mathrm{Wm}^{-2} \mathrm{~m}^{-1} \mathrm{sr}^{-1}$ (dashed) and $B_{\lambda}=3 * 10^{8} \mathrm{Wm}^{-2} \mathrm{~m}^{-1} \mathrm{sr}^{-1}$ (solid); $Q_{x}=10^{2}, 10^{1}, 10^{0}, 10^{-4}, 10^{-6}$ (red/blue/brown/violet/green respectively); $\alpha=0.1 \mathrm{~km}^{-1}$.

$$
r_{\max }=\sqrt{V \exp \left[-2 \alpha_{0} R_{0}\left(\frac{\alpha}{\alpha_{0}} r_{\max }-1\right)\right] U^{-1}}
$$

Results of estimations obtained for lidar with SiPM and PMT detectors are provided in Fig. 10. They show the spectral dependences of maximum achievable operation range $r_{\max }=f(\lambda)$ by the atmospheric lidar for a wide range of $Q_{x}$ parameter variations $\left(Q_{x}=10^{2}, 10^{1}, 10^{0}, 10^{-4}, 10^{-6}\right)$ at conditions of clean atmosphere with $\alpha=10^{-1} \mathrm{~km}^{-1}$ and a weak haze with $\alpha=1 \mathrm{~km}^{-1}$ and for two levels of daytime sky background: from an average brightness of $3 \cdot 10^{7}$ to its high level of $3 \cdot 10^{8} \mathrm{~W} \cdot \mathrm{m}^{-2} \cdot \mathrm{m}^{-1} \mathrm{sr}^{-1}$. We used the UPC lidar [20] with SiPM photodetectors SensL 30035 (SiPM-S) and the Hamamatsu S10362-33-100C (SiPM-H) at a given SNR with the receiving channel bandwidth: $\Delta f=300 \mathrm{MHz}$ and $20 \mathrm{MHz}$. For comparison, we used the PMT general (hereinafter: PMT-gen) as a generalized virtual PMT introduced earlier in [15].

As we are interested to know the extent of operation range reduction under strong sky background for SiPM detectors, by following [15] we can obtain the expression:

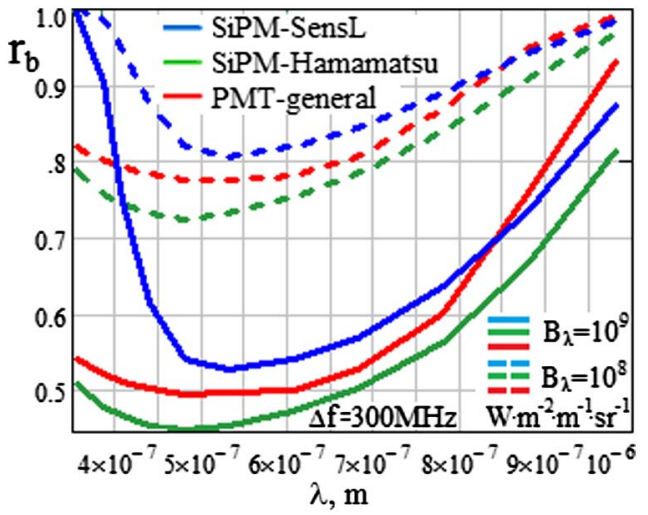

Fig. 11. $r_{b}$ as a function of $\lambda$ for $B_{\lambda}=10^{9}$ and $10^{8} \mathrm{~W} \mathrm{~m}^{-2} \mathrm{sr}^{-1} \mathrm{~m}^{-1}$; $\Delta f=300 \mathrm{MHz}$

photodetectors under consideration, we obtain the results shown in Figs. 11 and 12 .

As is seen from Figs. $\underline{11}$ and $\underline{12}$, the SiPM-H with a most sensitive $\eta(\lambda)$ curve of all three detectors, when following Eq. (16), in turn, is a most responsive one to the lidar operation range reduction. The PMT-gen is less responsive to the background influence because its $\eta(\lambda)$ curve lies considerably below than SiPMs

$$
r_{b} \equiv \frac{R_{B}}{R_{\text {ref }}}=\sqrt{\left[\sqrt{1+\frac{4}{\rho_{\text {out }}^{2}} \frac{P_{n}^{2}}{P_{q}^{2}}\left(1+\frac{P_{b} P_{q}}{P_{n}^{2}}\right)}-1\right] /\left[\left(1+\frac{P_{b} P_{q}}{P_{n}^{2}}\right)\left(\sqrt{1+\frac{4}{\rho_{\text {out }}^{2}} \frac{P_{n}^{2}}{P_{q}^{2}}}-1\right)\right]} .
$$

If we apply Eq. (15) to lidars with SiPMs and PMTs, when inequalities $P_{b} \gg P_{q} \gg P_{n}$ are satisfied, and then use $U_{i}$ from Eq. (10), we can obtain: $r_{b}=U_{i}^{-1 / 2}$, for specific receiver parameters and certain sky background:

$$
r_{b}=\sqrt[4]{2 h c \Delta f / \lambda} \cdot \sqrt[4]{F / \eta(\lambda)} / \sqrt[4]{P_{b}} .
$$

By applying all the specific features of the lidar system and assessments necessary to the silicon curves in the central part of a spectral range under consideration. The SiPM-S is in the intermediate position.

\section{Modified Parameterization Scheme of Multiwavelength} Lidar at Real Background Conditions

Let's recall that we are discussing a dimensionless parameterization of the lidar SNR [Eq. (1)] presented as the product of five independent dimensionless parameters, each of which has its own source. In 


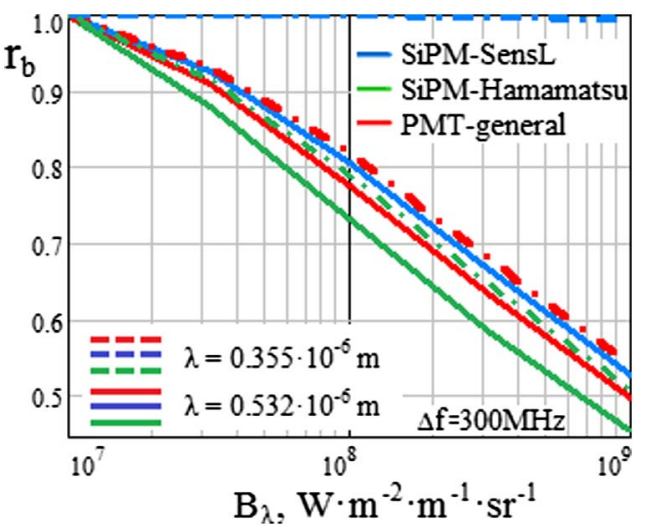

Fig. 12. $r_{b}$ as a function of $B_{\lambda}$ for $\lambda=355$ and $532 \mu \mathrm{m}$. $\Delta f=300 \mathrm{MHz}$.

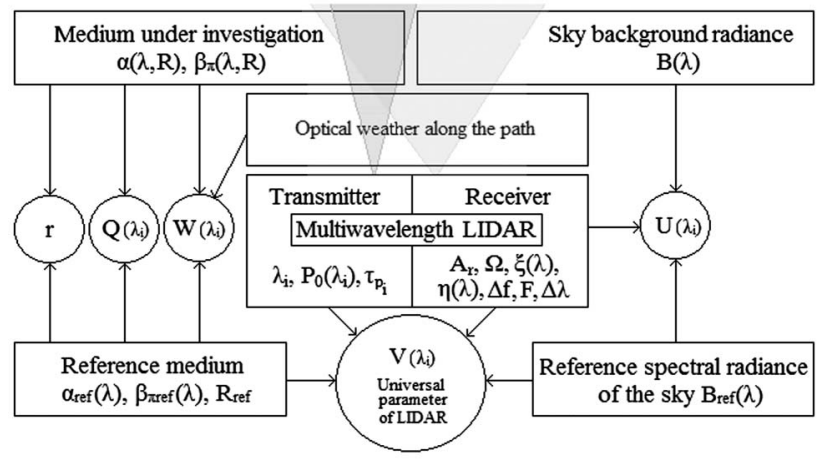

Fig. 13. Block diagram of a renewed dimensionless parameterization scheme for multiwavelength lidar.

Fig. 13, a block diagram of the updated model of dimensionless parameterization of multiwavelength lidar is shown.

It clearly points to a certain relationship between the normalized parameters and components of measurement system, including the atmosphere state and the daytime sky background, the lidar transmitter and receiver, the reference medium and reference background brightness.

\section{Conclusions}

As appears from the results obtained in the paper, in the development of our previous approaches, we considered the practical applicability of the $V$ parameter by extending it to all wavelengths of multiwavelength sensing LIDAR and by adapting it to reference level of the daytime sky background guided by a more generalized approach to estimate the threshold power. Our use of the background radiation's reference brightness within its usual range of changes allows us to evaluate the vulnerability of a particular lidar system worked at the number of wavelengths from impact of the background noise of moderate radiance and to predict reasonably the degree of deterioration of the lidar's detection threshold due to the impact of sky background of arbitrary radiance.
Joint account of the reference atmosphere and the reference sky background in dimensionless parameter $V\left(\lambda_{i}\right)$ allows us to characterize quantitatively the ability to sense the standard molecular atmosphere and to withstand exposure of the reference level external background, which is unavoidable in daytime conditions. This allows us to uniquely determine the energy potential of lidar at each sensing wavelength and to compare different lidars and predict their response, taking into account all the important parameters of their transceiver tract.

We have introduced a new principle of forming a $U$ parameter, which characterizes the degree of immunity of the lidar receiving system to background radiation environment. On the basis of newly introduced criteria, a set of parameters $U_{i}$ is represented as a ratio of the photodetector thresholds where current background noise and background noise are available with reference radiance and the solar spectrum accounted.

The results of the lidar measurements with SiPMs photodetectors operating in analog mode and photon counting show that, along with the traditional PMTs, they can be successfully used in lidar systems in all signal-detection modes. Real possibilities of implementing the photon-counting mode in the atmospheric lidar on SiPMs were demonstrated in practice for the first time as an important addition to the analog-mode detection, which significantly increases the lidar capabilities. In this case, the raw experimental data of lidar measurements can be subjected to traditional methods of algorithmic processing of lidar echoes.

Basing on the experimental studies with SiPMs as photodetectors for laser remote sensing, we have considered the applications of dimensionless parametrization model to relatively new lidars with SiPMs. The updated methods of forming the V-U-VUparameters have been applied to SiPM lidars, with analysis of capabilities of the multiwavelength lidars sounding at the range of $0.4-1.0 \mu \mathrm{m}$. To be more illustrative, it was done in comparison with traditional PMT lidars.

Following the renewed methodology, we have shown what does determine a value and what is in practice a range of lidar with two types of SiPMs in comparison with PMT photodetectors with accounting the most important parameters of the multiwavelength system. By using the renewed V-UVU-parameters, it has been demonstrated what is the lidar operation range reduction and what are the concentrations of gases/vapors measurable by SiPM lidar against the daytime sky background.

Combining and using new principles of $V$ and $U$ parameters definition illustrated by the updated block-diagram of dimensionless parameterization of multiwavelength lidars, allows all users to effectively describe and analyze any lidar systems by taking into account the relationships between the measuring system parameters, including the lidar transmitter and receiver, the standard atmosphere as a 
reference medium and a reference brightness of the background, as well as the actual state of the atmosphere and the current daytime sky background.

\section{Appendix A: Definition of Variables Used}

$A_{r} \quad$ receiver's area $\left[\mathrm{m}^{2}\right]$

$B_{\lambda} \quad$ sky background radiance at wavelength $\lambda$ $\left[\mathrm{W} \cdot \mathrm{m}^{-2} \mathrm{~m}^{-1} \mathrm{sr}^{-1}\right.$ ]

$B_{\text {ref } \lambda}$ referent sky background radiance at $\lambda$ $\left[\mathrm{W} \cdot \mathrm{m}^{-2} \mathrm{~m}^{-1} \mathrm{sr}^{-1}\right]$

$B_{\text {ref }}$ referent sky background radiance $\left[\mathrm{W} \cdot \mathrm{m}^{-2} \mathrm{~m}^{-1} \mathrm{sr}^{-1}\right]$

$c \quad$ light speed $[\mathrm{m} / \mathrm{s}]$

$F$ excess noise factor

$H$ Planck's constant [J s]

$K$ Boltzmann's constant [J/K]

NEP noise equivalent power $\left[\mathrm{W} / \mathrm{Hz}^{1 / 2}\right]$

$P_{0} \quad$ transmitter pulse power [W]

$P_{b} \quad$ power of background radiation [W]

$P_{n}$ internal noise power normalized to the input [W]

$P_{q} \quad$ quantum noise power [W]

$P_{s}, P_{s 0} \quad$ signal power and signal power received from the reference range [W]

$P_{t}, P_{t 0} \quad$ photodetector's threshold power in presence and absence of background radiation [W]

$P_{t B} \quad$ photodetector's threshold power with arbitrary background radiance [W] available

$Q_{B S}$ aerosol backscatter efficiency to the molecular reference

$Q_{X} \quad$ backscatter efficiency of arbitrary species to the molecular reference

$R, R_{\text {ref }}$ operation range and reference range [m]

$R_{B} \quad$ operation range under intense background [m]

$r$ normalized range factor

$r_{b}$

$r_{\max }$

$s_{\text {rel }}$

$S_{\lambda} \quad$ emissivity of sun spectrum $\left[\mathrm{W} / \mathrm{m}^{2} \cdot \mathrm{sr} \cdot \mathrm{m}\right]$

$T, T_{0}$ transparency and transparency of the standard molecular atmosphere

$U, U_{b}$ ratio of photodetector's threshold powers taken in presence and in absence of background radiation

$V$ ratio of echo-signal power $P_{s 0}$ received from reference range $R_{\text {ref }}$ of the reference molecular atmosphere to photo-detector threshold power $P_{t 0}$ in the absence (or at reference level) of background noise

$W$ normalized atmospheric component determined by transparencies ratio of the current atmosphere state and the standard molecular atmosphere

$\alpha_{a}, \alpha_{m}$ extinction coefficients in aerosol and molecular atmospheres $\left[\mathrm{m}^{-1}\right]$

$\beta_{a}, \beta_{m}$ backscattering coefficients in aerosol and molecular atmospheres $\left[\mathrm{m}^{-1}\right]$

$\begin{aligned} \beta_{\pi} & \text { backscattering coefficient }\left[\mathrm{m}^{-1}\right] \\ \Delta_{f} & \text { receiver transmission band [Hz] } \\ \Delta_{\lambda} & \text { interference filter bandwidth [m] } \\ \eta(\lambda) & \text { photon detection efficiency curve of photo- } \\ & \text { detector } \\ \lambda, \lambda_{\text {ref }} & \text { wavelength and reference wavelength [m] } \\ \xi & \text { optics' transmission } \\ \rho & \text { signal-to-noise ratio } \\ \rho_{\text {out }} & \text { output signal-to-noise ratio } \\ \tau_{p} & \text { pulse duration [s] } \\ \Psi_{s} & \text { normalized signal power or signal power } \\ & \text { normalized to quantum noise } \\ \Psi_{b} & \text { normalized background noise power or } \\ & \text { background noise power normalized to } \\ \Psi_{n} & \text { quantum noise } \\ & \text { normalized internal noise power or internal } \\ \Psi_{t} & \begin{array}{l}\text { noise power normalized threshold signal power or } \\ \text { threshold signal power normalized to quan- }\end{array} \\ & \text { tum noise } \\ \Psi_{X} & \text { signal-to-noise ratio at lidar photodetector } \\ & \text { input } \\ \Omega & \text { field of view [sr] }\end{aligned}$

The authors thank the Spanish Ministry of Science and Innovation (MICINN) for projects DPI200913379, and DPI2011-25525, which partially funded this research. The lidar activities are supported by the EU's 7th FP project «Aerosols, Clouds, and Trace Gases Research Infrastructure Network (ACTRIS)», grant agreement 262254, and by the MICINN, Ministry of Economy and Competitiveness and FEDER funds under the projects TEC2009-09106/TEC and TEC2012-34575, and the Complementary Actions CGL2009-08031-E/CLI， CGL2010-09225-E， CGL2011-13580-E/CLI, and CGL2011-16124-E/CLI.

\section{References}

1. R. Agishev, Lidar Monitoring of the Atmosphere (PhysMathLit, 2009).

2. R. Agishev, Protection from Background Clutter in ElectroOptical Systems of Atmosphere Monitoring (Mashinostroenie Publishing House, 1994).

3. D. Killinger, Lidar and Laser Remote Sensing: Handbook of Vibrational Spectroscopy (Wiley, 2002).

4. V. Kovalev and W. E. Eichinger, Elastic Lidar: Theory, Practice, and Analysis Methods (Wiley-Interscience, 2004).

5. T. Leblanc, T. Trickl, and H. Vogelmann, "Lidar," in Monitoring Atmospheric Water Vapour: Ground-Based Remote Sensing and In-situ Methods, N. Kampfer, ed. (Springer, 2009), pp. $113-158$.

6. C. Weitkamp, ed., Lidar: Range-Resolved Optical Remote Sensing of the Atmosphere (Springer, 2005).

7. J. Minkoff, Signal Processing Fundamentals and Applications for Communications and Sensing Systems (Artech House, 2002).

8. V. Molebny, G. Kamerman, and O. Steinvall, "Laser remote sensing: yesterday, today and tomorrow," Electron. Commun. 3, 68-73 (2011)

9. G. Osche, Optical Detection Theory for Laser Applications (Wiley, 2002).

10. W. Grant, E. V. Browell, R. T. Menzies, K. Sassen, C. Y. She, and C.-Y. She, eds., Selected Papers on Laser Applications in Remote Sensing (SPIE, 1997). 
11. W. Wiegner, "Lidar for aerosol remote sensing," in Atmospheric Physics: Background, Methods, Trends, U. Schumann, ed. (Springer-Verlag, 2012) pp. 449-464.

12. R. Measures, Laser Remote Sensing: Fundamentals and Applications (Wiley, 1994).

13. R. Agishev, A. Comeron, B. Gross, F. Moshary, S. Ahmed, and A. Gilerson, "Application of the method of decomposition of lidar signal-to-noise ratio to the assessment of laser instruments for gaseous pollution detection," Appl. Phys. B 79, 255-264 (2004).

14. R. Agishev and A. Comeron, "Spatial filtering efficiency of biaxial monostatic lidar: analysis and applications," Appl. Opt. 41, 7516-7521 (2002).

15. R. Agishev, B. Gross, F. Moshary, S. Ahmed, and A. Gilerson, "Simple approach to predict APD/PMT lidar detector performance under sky background using dimensionless parameterization," Opt. Lasers Eng. 44, 779-796 (2006).

16. R. Agishev, B. Gross, F. Moshary, S. Ahmed, and A. Gilerson, "Development of a SNR parameterization scheme for general lidar assessment," Appl. Phys. B 80, 765-776 (2005).

17. M. Ross, Laser Receivers (Wiley, 1966).

18. G. Barbarino, R. de Asmundis, and G. de Rosa, "Silicon photo multipliers detectors operating in Geiger regime," in Photodiodes-World Activities in 2011, J.-W. Park, ed. (InTechOpen, 2011), pp. 183-226.

19. A. Del Guerra, N. Belcari, M. Bisogni, and F. Corsi, "Silicon photomultipliers as novel photodetectors for PET," Nucl. Instrum. Methods Phys. Res. A 648, 232-235 (2011).

20. J. Riu, M. Sicard, S. Royo, and A. Comeron, "Silicon photomultiplier detector for atmospheric lidar applications," Opt. Lett. 37, 1229-1231 (2012). 